\title{
On the definition of superclusters
}

\author{
Gayoung Chon ${ }^{1}$, Hans Böhringer ${ }^{1}$, and Saleem Zaroubi ${ }^{2}$ \\ ${ }^{1}$ Max-Planck-Institut für extraterrestrische Physik, Giessenbachstrasse, 85748 Garching, Germany \\ e-mail: gchon@mpe.mpg.de \\ 2 Kapteyn Astronomical Institute, University of Groningen, PO Box 800, 9700 AV Groningen, The Netherlands \\ Received 27 December 2014 / Accepted 6 February 2015
}

\section{ABSTRACT}

\begin{abstract}
To obtain a physically well-motivated definition of superclusters, we proposed in our previous work to select superclusters with an overdensity criterion that selects only those objects that will collapse in the future, including those that are at a turn-around in the present epoch. In this paper we present numerical values for these criteria for a range of standard cosmological models. We express these criteria in terms of a density ratio or, alternatively, as an infall velocity and show that these two criteria give almost identical results. To better illustrate the implications of this definition, we applied our criteria to some prominent structures in the local Universe, the Local supercluster, Shapley supercluster, and the recently reported Laniakea supercluster to understand their future evolution. We find that for the Local and Shapley superclusters, only the central regions will collapse in the future, while Laniakea does not constitute a significant overdensity and will disperse in the future. Finally, we suggest that those superclusters that will survive the accelerating cosmic expansion and collapse in the future be called "superstes-clusters", where "superstes" means survivor in Latin, to distinguish them from traditional superclusters.
\end{abstract}

Key words. large-scale structure of Universe - X-rays: galaxies: clusters

\section{Introduction}

Superclusters are the largest prominent density enhancements in our Universe. In the framework of hierarchical structure formation, superclusters are the next objects up from clusters, but unlike clusters, they are not virialised. They are generally defined as groups of two or more galaxy clusters above a certain spatial density enhancement (Bahcall 1988). In this sense superclusters have mostly been treated just as a collection of clusters. Without a clear definition, we are left with structures with heterogeneous properties. Unlike clusters, superclusters have not reached a quasi-equilibrium configuration that defines their structure. As we observe them today, they are transition objects that largely reflect their initial conditions. In contrast, clusters can be approximately described by their equilibrium configuration, as given by, for example, the NFW model (Navarro et al. 1995). Even disturbed and merging clusters are characterised by their deviations from this model.

For transition objects like superclusters, such a description is not possible. One solution to this problem is to include the future evolution in the definition of the object, selecting only those superclusters that will collapse in the future in a more homogeneous class of objects. There have been attempts to explore this definition in simulations (Dünner et al. 2006). We have been exploring a similar approach observationally in our construction of an X-ray supercluster catalogue (Chon et al. 2013, 2014). We selected the superclusters from the X-ray galaxy cluster distribution by means of a friends-of-friends (FoF) algorithm in such a way that we expect the superclusters to have their major parts gravitationally bound and to collapse in the future. We found that we obtain a good understanding of the properties of our supercluster sample, and we can recover many of the known superclusters described in the literature in our survey volume.

This selection is found to be slightly more conservative by not linking all the surrounding structure to the superclusters, which are linked to these objects in some other works. In other cases, such as for the Shapley supercluster, large structures are split into substructures. But overall the sample has good properties. Thus we find our method not only physically well motivated, but also appealing in selecting the structures that appear observationally distinct and prominent. To distinguish objects selected by our definition from the general usage of the word superclusters, we suggest that those systems be called "superstesclusters", relating to the Latin word superstes, which means survivor. Considering origins, superclusters were first studied at the time when most cosmologists favoured a marginally closed Universe in which all overdense regions would eventually collapse. It came with the general acceptance of a re-accelerating Universe that this concept of future collapse needed to be revised. We prefer using a new term over redefining the word supercluster, out of respect for the previous studies that were done with a less strict definition.

It is our goal with this paper to explore this definition of superclusters and its consequences in some more detail. In particular, we provide numerical values for the selection criteria for various cosmologies. So far, we have based our selection criterion on the matter overdensity, which is motivated by our X-ray cluster observations. In theory, on large scales where the dynamics is dominated by gravity, observations of velocity fields should closely reflect the dynamical evolution of structures and the underlying mass distribution. The velocity field also does not suffer from the bias that clusters and, to an extent, galaxies have. As a result, the dynamical information may provide a better basis for predicting the future evolution of the large-scale structure (Zel'dovich 1970; Shandarin \& Zeldovich 1989; Dekel 1994; Zaroubi et al. 1999). Therefore we also explore the selection criteria in terms of the infall velocity. In our case we expect a close correspondence between the overdensity and the streaming motions, since the large-scale structure at the scale of superclusters is still in the quasi-linear regime of structure formation. 
Nevertheless, we test the correspondence between the two descriptions in the paper.

We lay out our concept and explain the criterion in Sect. 2. In Sect. 3 we illustrate the concept by three applications and Sect. 4 provides discussion and summary.

\section{Theoretical concept}

To study which primordial overdensities will finally collapse, we approximate the overdense regions by spheres with homogeneous density. This approximation has been successfully used for many similar investigations. We can then model the evolution of the overdensity with respect to the expansion of the background cosmology with reference to Birkhoff's theorem. This allows us to describe the evolution of both regions, overdensity and background, by the respective values of the local and global parameters of Hubble constant, $H$, matter density, $\Omega_{\mathrm{m}}$, and the parameter corresponding to the cosmological constant, $\Omega_{\Lambda}$.

We calculate the evolution of the local and the global regions by integrating the Friedmann equation for their dynamical evolution. We use $z=500$ as the starting point of our integration, since we have found that with this starting redshift, the final results have an accuracy well below $1 \%$. To find the starting values, we find the relevant cosmological parameters mentioned above for the background cosmology at $z=500$. We then define an overdense region by increasing $\Omega_{\mathrm{m}}$ so that we find a collapsed region in the future, which is solved iteratively.

In comparison with the structures seen today, we are interested in the following properties of these marginally collapsing objects, which should be observable. What are their typical matter overdensities in the current epoch? What is the Hubble parameter that characterises their current, local dynamical evolution? These parameters will depend on the characteristics of the background cosmology. We have therefore calculated the density and expansion parameters for collapsing overdensities in a set of relevant cosmologies. We assumed a flat cosmology, with $\Omega_{\mathrm{m}}+\Omega_{\Lambda}=1$ for most models with the exception of one example for an open cosmology. Amongst the models shown are the bestfit results from the Planck (Planck Collaboration XVI 2014) and WMAP (Hinshaw et al. 2013) missions.

The parameters shown in Table A.1 are the density ratio of the overdensity to the background density, $R=\rho_{\mathrm{ov}} / \rho_{\mathrm{m}}$, the parameter characterising the homogeneous expansion of the overdense sphere given in form of a Hubble parameter, $\mathrm{H}_{\mathrm{ov}}$, and the overdensity of the local region with respect to the critical density, $\rho_{\mathrm{c}}$, of the Universe, $\Delta_{\mathrm{c}}=\left(\rho_{\mathrm{ov}}-\rho_{\mathrm{c}}\right) / \rho_{\mathrm{c}}$. The results show that while the density ratio varies with the matter density in the background universe, the overdensity parameter with respect to critical density hardly changes. Changing the Hubble constant does not alter the nature of the solution. The parameters, $R$ and $\Delta_{\mathrm{c}}$, stay constant, while the local expansion parameter, $\mathrm{H}_{\mathrm{ov}}$, only scales with the Hubble parameter of the background cosmology. Therefore we show only one example for the change in the parameters with the Hubble constant.

Another interesting characterisation for superclusters are those structures that are at turn-around now. These structures have decoupled from the Hubble flow already and are at rest in the Eulerian reference frame so are just starting to collapse now. Here the local Hubble parameter is zero by definition. The Einstein-de Sitter (EdS) model with the parameter, $\Omega_{\mathrm{m}}=1.0$, yields a value of $\Delta_{c}=(3 \pi / 4)^{2}-1 \sim 4.55$, which can also be calculated analytically.

\subsection{Comparison of overdensity and dynamical criteria}

The threshold parameters for collapse given in Table A.1 are only valid for spherically symmetric overdensities, which are reasonable approximations for realistic overdensities. As described in the previous section, the velocity field provides a better basis for predicting the future evolution of a large-scale structure than does the density distribution (Dekel 1994; Zaroubi et al. 1999). Also in observations, the density distribution of objects has to be corrected for their large-scale structure bias, which is not necessary for evaluating the velocity field. However, in current astronomical observations, peculiar velocity data are only available for the very local region of the Universe, and for most other applications, we only have estimates of overdensity. Therefore it is important to test how well our criteria that are based on the overdensity argument correspond to those on the velocity information for realistic supercluster morphologies.

For this reason we used the cosmological $N$-body simulations (Springel et al. 2005) to compare the radius inside which the structure is predicted to collapse based on the overdensity, $r_{\Delta}$, to that of the infall velocity, $r_{v}$ for 570 superstes-clusters. For the details of the construction of the superclusters in simulations, we refer the readers to Chon et al. (2014). The value of $r_{\Delta}$ was taken at the radius where the density ratio reaches the threshold value for collapse, and $r_{v}$ is defined as the radius where the required infall velocity is reached. The latter radius marks the largest distance within which, on average, the infall velocities of all haloes are detached from the Hubble flow with the local expansion parameter prescribed in the previous section. The very close correspondence between the two predictions are shown in Fig. A.1.

We only have five pathological cases, where the collapse overdensity is only reached once away from the centre, while the infall pattern never reaches the required threshold. These are the cases where the most massive structures are concentrated not at the centre of the supercluster, but near the radius, $r_{v}$, and beyond. In these cases the velocity pattern is very different from a smooth radial infall, and the supercluster is most probably fragmented into two or more massive substructures near the supercluster boundary in the future. We find it as a very strong encouragement for our approach, where the two alternative criteria usually give very similar results. The good correspondence is also a confirmation that structure evolution on the scale of superclusters is still in the quasi-linear regime.

\section{Applications}

In this section we illustrate the implications of our superstescluster definition with respect to some of the known superclusters. The homogeneous sphere approximation only gives a rough estimate of the collapse situation. More detailed solutions have to take the morphology and substructure of the systems into account, which has actually been done for some of the cases below. Both criteria listed in Table A.1, the overdensity and the peculiar infall velocity, can be used for such a first estimate. The aim of the following discussion is therefore only an approximate application of the suggested criteria for illustration. We use parameters for the cosmological model with $\Omega_{\mathrm{m}}=0.3$ and $H_{0}=70 \mathrm{~km} \mathrm{~s}^{-1} \mathrm{Mpc}^{-1}$ for the considerations below.

\subsection{Local supercluster and Virgo infall}

The Local supercluster is a high concentration of matter roughly centred on the Virgo cluster, which includes the Milky Way and the Local Group in the outskirts. It was first described by 
de Vaucouleurs $(1953,1958)$. Detailed studies found the system to be mostly concentrated in an elongated filament that extends about $40 h^{-1} \mathrm{Mpc}$, e.g. Tully \& Fisher (1978), Karachentsev \& Makarov (1996), Lahav et al. (2000), Klypin et al. (2003). The Local Group is at a distance of about 16 to $17 \mathrm{Mpc}$ from Virgo, and it shows a peculiar infall velocity towards the Virgo cluster of about $220 \pm 30 \mathrm{~km} \mathrm{~s}^{-1}$ (Sandage et al. 2010) or $\sim 250 \mathrm{~km} \mathrm{~s}^{-1}$ (Klypin et al. 2003).

Applying the peculiar velocity criterion for future collapse, we find the following. The necessary peculiar infall velocity at a certain distance from the centre of the supercluster is given by the difference of the local to the background Hubble parameters multiplied by the distance, in our case $d \times 49.5 \mathrm{~km} \mathrm{~s}^{-1} \mathrm{Mpc}^{-1}$. Thus at the distance of the Local Group, a peculiar infall velocity of about $800 \mathrm{~km} \mathrm{~s}^{-1}$ would be required. Therefore the Local Group will recede from the Local supercluster in the distant future. This has been concluded in several previous works, and it has been shown, for example, by $N$-body simulations by Nagamine \& Loeb (2003) based on a numerical constraint reconstruction of the local Universe by Mathis et al. (2002). Inspecting the velocity flow patterns of the Local supercluster shown in Klypin et al. (2003), Tully \& Mohayaee (2004), and Courtois \& Tully (2012), we find that only inside $10 \mathrm{Mpc}$ infall velocities up to $500 \mathrm{~km} \mathrm{~s}^{-1}$ seem to occur, reaching the lower limit for a collapse. We can also use the estimate of the infall velocity profile from the constraint reconstruction of the Local supercluster by Klypin et al. (2003) to find the outermost collapsing shell of the supercluster. They approximate the mean infall pattern by $v=145\left(13 h^{-1} \mathrm{Mpc} / r\right)^{1 / 2} \mathrm{~km} \mathrm{~s}^{-1}$. With this prescription we find that only the regions inside about $5.5 \mathrm{Mpc}$ will collapse in the future.

We can also use the spherical collapse model to obtain a mass estimate of the Virgo cluster and its surroundings from the peculiar velocity of the Milky way towards the Virgo cluster of $\sim 250 \mathrm{~km} \mathrm{~s}^{-1}$. When assuming a Virgo distance of $16 \mathrm{Mpc}$ (Tonry 1991), the infall peculiar velocity corresponds to a local Hubble parameter of $\sim 54.5 \mathrm{~km} \mathrm{~s}^{-1} \mathrm{Mpc}^{-1}$ for a homogeneous sphere with its centre at the Virgo distance. An integration of the Friedmann equations for our fiducial cosmology infers a ratio of the local overdensity to the cosmic mean of about 2.6. This translates into a mass of Virgo and surroundings inside a radius of $16 \mathrm{Mpc}$ of $1.8 \times 10^{15} M_{\odot}$. Tully \& Mohayaee (2004) get a mass of $1.2 \times 10^{15} M_{\odot}$ from fitting the infall pattern, which probably gives most weight to the measurement at a slightly smaller radius. Klypin et al. (2003) obtain a mass of $\sim 10^{15} M_{\odot}$ for Virgo and the central filament of the Local supercluster from a constrained reconstruction of the Local supercluster. Karachentsev et al. (2014) studied the infall pattern of tracers with good distance estimates from the HST observations, and find a mass of $8 \times 10^{14} M_{\odot}$ inside a radius of $7.2 \mathrm{Mpc}$ which they estimate to be the turn-around radius at the current epoch. The fair agreement of the different methods shows that the spherical infall models provide an excellent first estimate of the fate of such a supercluster.

\subsection{Laniakea}

Based on the compilation of peculiar velocities of galaxies out to $z=0.1$ in Tully et al. $(2013,2014)$ presented a new supercluster, which they call Laniakea. We refer the readers to Tully et al. (2013) for the detailed methodology, but in essence they rely on the absolute distance measures estimated from six methods including the Tully-Fisher relation to calculate peculiar velocities of galaxies within $400 h^{-1} \mathrm{Mpc}$, where the coverage is sparse beyond $100 \mathrm{~h}^{-1} \mathrm{Mpc}$. In total they report distance measures for more than 8000 galaxies in the whole survey region. To reconstruct the underlying velocity field, they used the Wiener filter algorithm (Zaroubi et al. 1995). They conclude from the reconstructed velocity field that there is a coherent flow within a sphere of radius, $80 \mathrm{~h}^{-1} \mathrm{Mpc}$, which contains an estimated mass of $10^{17} h^{-1} M_{\odot}$, and they define this region as a supercluster. Their mass estimate implies that the ratio of Laniakea density to the mean density is about 0.94 . Thus Laniakea does not even constitute a region with a significant overdensity and does not fulfil our criteria in Table A.1 for a supercluster. As a result, Laniakea is far from being a bound system, and as a whole, it will disperse in the future, while only several dense regions will collapse within.

We can also look at Laniakea from another point of view by applying the peculiar velocity argument. If Laniakea was a bound structure with a radius of $80 h^{-1} \mathrm{Mpc}$, we would estimate the required infall velocity at the boundary to be about $5700 \mathrm{~km} \mathrm{~s}^{-1}$ based on the local Hubble parameter estimate given in Table A.1 for future collapse. However, typical peculiar velocities in their catalogue hardly exceed $500-700 \mathrm{~km} \mathrm{~s}^{-1}$ in the survey. This emphasises again that Laniakea as a whole is not a bound structure.

We also note that their value of the Hubble constant obtained by minimising the velocity monopole is $75.2 \pm$ $3.0 \mathrm{~km} \mathrm{~s}^{-1} \mathrm{Mpc}^{-1}$. There are also other local measurements of $H_{0}$, notably by Riess et al. (2011) based on 253 Type Ia supernovae data from the HST and by Freedman et al. (2012) based on an additional mid-infrared observation of Cepheids. The former gives $H_{0}$ of $74.8 \pm 3.1$, and the latter $74.3 \pm 2.1 \mathrm{~km} \mathrm{~s}^{-1} \mathrm{Mpc}^{-1}$, both with less than $3 \%$ uncertainty. Within their respective errors, the three measurements agree, which is also pointed out by Tully et al. (2014). It is interesting to compare these values to $H_{0}$ measured by the cosmic microwave background (CMB) experiments, WMAP, and Planck, where $H_{0}$ is more sensitive to very large scales. The best-fit $H_{0}$ values are $70.0 \pm 2.2$ (Hinshaw et al. 2013) and $68.0 \pm 1.4 \mathrm{~km} \mathrm{~s}^{-1} \mathrm{Mpc}^{-1}$ (Planck Collaboration XVI 2014), so local measurements of $H_{0}$ are always greater than those from the CMB measurements, although they would agree within their respective current $1 \sigma$ uncertainty. With this it is interesting to note that Turner et al. (1992) pointed out that a locally underdense Universe would yield a Hubble constant larger than the cosmic mean. In reference to our work (Böhringer et al. 2015), we find a local region within a radius of $170 \mathrm{~h}^{-1} \mathrm{Mpc}$ in the southern sky, which is under-dense by $\approx 20 \%$ with respect to the mean density resulting from the cluster number density that is under-dense by $40 \%$ with a cluster bias of about two. Since the REFLEX survey has a high completeness up to a redshift of $z \sim 0.3$, we have a good handle for tracing the matter density out to a very large radius, containing the volume of Laniakea. The amplitude of the local under-density traced by REFLEX II of $\approx 20 \%$, which implies that the locally measured $H_{0}$ would be $3 \pm 1.5 \%$ more than for the cosmic one. It therefore indicates that the $H_{0}$ value obtained by Tully et al. (2014) is consistent with the case where $H_{0}$ is measured in an under-dense region.

\subsection{Shapley supercluster}

The Shapley supercluster is known to have the highest concentration of galaxies in the nearby Universe, at a redshift around 0.046 (Scaramella et al. 1989; Raychaudhury 1989). X-ray emission in the central region of Shapley was first mapped by Kull \& Böhringer (1999), and it even traces the intra-cluster gas. We also report three X-ray superclusters in the area of the Shapley 
supercluster, constructed with a cluster overdensity parameter, $f=10$, from the REFLEX II cluster catalogue (Chon et al. 2013). Here, $f$ is related to the density ratio $R$ by, $f=(R-$ 1) $b_{\mathrm{CL}}+1$ where $b_{\mathrm{CL}}$ is a cluster bias factor. Even with a generous choice of $f=10$, this already indicates that Shapley will be broken into smaller concentrations of matter, rather than collapsing into one large object. To get deeper insight, we calculated density ratios with data taken from the literature and from our X-ray work described below.

Reisenegger et al. (2000) used velocity caustics from 3000 galaxies to define a central region of Shapley and estimated the upper bound mass in a spherical radius of $8 h^{-1} \mathrm{Mpc}$ to be $1.3 \times 10^{16} h^{-1} M_{\odot}$. In this case the density ratio, $R$, is 20.7. Ragone et al. (2006) established a mass function of about 180 systems with redshifts in the Shapley region and give a lower mass limit of $1.1 \times 10^{16} h^{-1} M_{\odot}$ within the same volume as above. In this case, $R$ is about 17.5. Both results indicate that the central $8 h^{-1} \mathrm{Mpc}$ of the Shapley supercluster is likely to collapse into a more massive system. In fact, the density ratios are higher than required for a turn-around, which now implies that the very central region of Shapley supercluster has already started to collapse. To evaluate the total mass of Shapley with the REFLEX II clusters, we also considered the total cluster mass as a function of a radius for the same volume. We converted the total cluster mass to the total supercluster mass by adopting a scaling relation found with cosmological $N$-body simulations in Chon et al. (2014). We find that the required density ratio for collapse is satisfied out to about $12.4 \mathrm{~h}^{-1} \mathrm{Mpc}$. The derived total mass of Shapley within this distance is $1.34 \times 10^{16} h^{-1} M_{\odot}$. In fact, the turn-around density ratio is already reached at $11.1 h^{-1} \mathrm{Mpc}$, which is consistent with the density ratios estimated from previous work. We also note that the filamentary X-ray emission shown in Fig. 2 of Kull \& Böhringer (1999) coincides with the core of an already collapsing part of Shapley. The estimates of the density ratios of Shapley therefore provide a consistent picture that the central $11 h^{-1} \mathrm{Mpc}$ is undergoing a collapse meaning that only a central part of Shapley supercluster will form a supercluster in the future even if the outskirts of Shapley are also rich in clusters.

\section{Discussion and summary}

In this paper we have emphasised the need for a clearer, more physically motivated definition of superclusters. We have shown that defining superstes-clusters as those objects that will collapse in the future leads to a conservative selection criterion that does not accept all objects that have been called superclusters in the literature, but it leads to a more homogeneous class of objects as seen in our previous work on supercluster construction.

Our superstes-cluster definition is also interesting for another reason. With this definition, we are selecting the most massive virialised objects that will form in the future. In Fig. A.1 we can, for example, identify the most massive structure in the Millennium simulation that will form a virialised dark matter halo in the future with the uppermost point in the plot. It has a collapse radius, $r_{v}$ of $17.3 h^{-1} \mathrm{Mpc}$ and a corresponding mass of $1.94 \times 10^{16} M_{\odot}$. This can be compared to the collapsing fraction of the Shapley supercluster estimated in Sect. 3.3, which is quite comparable. Shapley is the highest mass concentration found in the nearby Universe in a volume that is quite similar to that of the Millennium simulation.

We find it very encouraging that similar mass estimates are obtained for the most massive structure in the observation and and in the simulation with our criteria for selecting superclusters. We have been applying this criterion in the construction of superstes-clusters using a FoF algorithm with a linking length tuned to select overdense regions that are close to collapse in the future. In our previous study (Chon et al. 2014) we found a close correspondence of the results of this method with the desired overdensity. It is, however, not one-to-one, and in particular, we find outliers for very large supercluster sizes, which are selected by the FoF algorithm but do not reach the overdensity threshold. In these extreme cases, superclusters appear as rather elongated filements, and the region that is bound to collapse is overestimated by the FoF-based method.

We studied the application of our definition to the Local supercluster and Shapley supercluster, as well as Laniakea. We find that the first two superclusters will collapse in the central regions while their outskirts are not gravitationally bound. For Laniakea, we find that this structure does not constitute a region with a significant overdensity and thus it cannot collapse as a whole. While the velocity structure described by Tully et al. (2014) highlights an impressively large structure in the local Universe, we feel that its labelling as a supercluster is not appropriate given that the region is not even overdense. It is interesting that Tully et al. (2014) and other surveys find a local Hubble parameter in this region that tends to be higher than the Hubble parameter measured on a more global scale (Planck Collaboration XVI 2014; Hinshaw et al. 2013). This can be taken as an indication that this region in the local Universe may be rather under-dense. This conclusion is supported by our recent studies with X-ray galaxy clusters and studies of the galaxy distribution (see Böhringer et al. 2015; Whitbourn \& Shanks 2014).

Acknowledgements. We thank the referee for the useful comments. H.B. acknowledges support from the DfG Transregio Programme TR33 and the DFG cluster of excellence "Origin and Structure of the Universe" (www . universe-cluster.de). G.C. acknowledges the support from the Deutsches Zentrum für Luft- und Raumfahrt (DLR) with the programme 50 OR 1403. S.Z. would like to acknowledge the support of The Netherlands Organisation for Scientific Research (NWO) VICI grant and Munich Institute for Astro- and Particle Physics (MIAPP) of the DFG cluster of excellence.

\section{Appendix A}

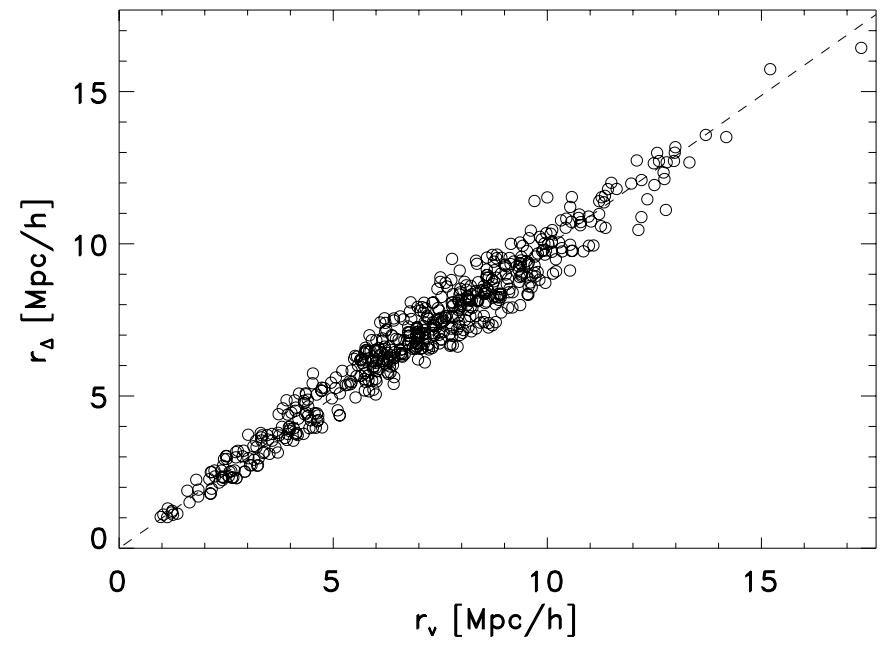

Fig. A.1. Comparison of the two radii that define the collapsing region of a supercluster. $r_{\Delta}$ is determined by the required density ratio criteria, and $r_{v}$ by the infall velocity criteria. The dashed line indicates the oneto-one line. 
G. Chon et al.: On the definition of superclusters

Table A.1. Present-epoch parameters characterising marginally collapsing objects in comparison to those at turn-around for various cosmological models. See text for an explanation of the listed parameters.

\begin{tabular}{cccccccc}
\hline \hline Label & $\Omega_{\mathrm{m}}$ & $h$ & $\begin{array}{c}R \\
\text { future collapse }\end{array}$ & $H_{\mathrm{ov}}$ & $\Delta_{\mathrm{c}}$ & $\begin{array}{c}R \\
\text { at turn-around }\end{array}$ & $\Delta_{\mathrm{c}}$ \\
\hline- & 0.24 & 0.7 & 9.811 & 17.60 & 1.36 & 14.31 & 2.43 \\
- & 0.27 & 0.7 & 8.732 & 19.09 & 1.36 & 13.12 & 2.54 \\
- & 0.30 & 0.7 & 7.858 & 20.55 & 1.36 & 12.15 & 2.65 \\
- & 0.33 & 0.7 & 7.134 & 22.00 & 1.35 & 11.35 & 2.75 \\
$\mathrm{WMAP}^{a}$ & 0.288 & 0.697 & 8.188 & 19.89 & 1.36 & 12.52 & 2.60 \\
Planck & 0.318 & 0.670 & 7.409 & 20.50 & 1.36 & 11.66 & 2.71 \\
- & 0.27 & 0.60 & 8.732 & 16.36 & 1.36 & 13.12 & 2.54 \\
EdS & 1.0 & 0.7 & - & - & - & 5.54 & 4.54 \\
open & 0.3 & 0.7 & 2.87 & 52.70 & & 12.48 & \\
\hline
\end{tabular}

Notes. ${ }^{(a)}$ Best fit for the combined model in Hinshaw et al. (2013); ${ }^{(b)}$ best fit for Planck and WMAP polarisation data (Planck Collaboration XVI 2014).

\section{References}

Bahcall, N. A. 1988, ARA\&A, 26, 631

Böhringer, H., Chon, G., Bristow, M., \& Collins, C. A. 2015, A\&A, 574, A26

Chon, G., Böhringer, H., \& Nowak, N. 2013, MNRAS, 429, 3272

Chon, G., Böhringer, H., Collins, C. A., \& Krause, M. 2014, A\&A, 567, A144

Courtois, H. M., \& Tully, R. B. 2012, Astron. Nachr., 333, 436

de Vaucouleurs, G. 1953, AJ, 58, 30

de Vaucouleurs, G. 1958, Nature, 182, 1478

Dekel, A. 1994, ARA\&A, 32, 371

Dünner, R., Araya, P. A., Meza, A., \& Reisenegger, A. 2006, MNRAS, 366, 803

Freedman, W. L., Madore, B. F., Scowcroft, V., et al. 2012, ApJ, 758, 24

Hinshaw, G., Larson, D., Komatsu, E., et al. 2013, ApJS, 208, 19

Karachentsev, I. D., \& Makarov, D. A. 1996, AJ, 111, 794

Karachentsev, I. D., Tully, R. B., Wu, P.-F., Shaya, E. J., \& Dolphin, A. E. 2014, ApJ, 782, 4

Klypin, A., Hoffman, Y., Kravtsov, A. V., \& Gottlöber, S. 2003, ApJ, 596, 19 Kull, A., \& Böhringer, H. 1999, A\&A, 341, 23

Lahav, O., Santiago, B. X., Webster, A. M., et al. 2000, MNRAS, 312, 166

Mathis, H., Lemson, G., Springel, V., et al. 2002, MNRAS, 333, 739

Nagamine, K., \& Loeb, A. 2003, New Astron., 8, 439
Navarro, J. F., Frenk, C. S., \& White, S. D. M. 1995, MNRAS, 275, 56

Planck Collaboration XVI. 2014, A\&A, 571, A16

Ragone, C. J., Muriel, H., Proust, D., Reisenegger, A., \& Quintana, H. 2006, A\&A, 445,819

Raychaudhury, S. 1989, Nature, 342, 251

Reisenegger, A., Quintana, H., Carrasco, E. R., \& Maze, J. 2000, AJ, 120, 523

Riess, A. G., Macri, L., Casertano, S., et al. 2011, ApJ, 730, 119

Sandage, A., Reindl, B., \& Tammann, G. A. 2010, ApJ, 714, 1441

Scaramella, R., Baiesi-Pillastrini, G., Chincarini, G., Vettolani, G., \& Zamorani, G. 1989, Nature, 338, 562

Shandarin, S. F. \& Zeldovich, Y. B. 1989, Rev. Mod. Phys., 61, 185

Springel, V., White, S. D. M., Jenkins, A., et al. 2005, Nature, 435, 629

Tonry, J. L. 1991, ApJ, 373, L1

Tully, R. B., \& Fisher, J. R. 1978, in Large Scale Structures in the Universe, eds. M. S. Longair, \& J. Einasto, IAU Symp., 79, 214

Tully, R. B., \& Mohayaee, R. 2004, in Outskirts of Galaxy Clusters: Intense Life in the Suburbs, ed. A. Diaferio, IAU Colloq., 195, 205

Tully, R. B., Courtois, H. M., Dolphin, A. E., et al. 2013, AJ, 146, 86

Tully, R. B., Courtois, H., Hoffman, Y., \& Pomarède, D. 2014, Nature, 513, 71

Turner, E. L., Cen, R., \& Ostriker, J. P. 1992, AJ, 103, 1427

Whitbourn, J. R., \& Shanks, T. 2014, MNRAS, 437, 2146

Zaroubi, S., Hoffman, Y., Fisher, K. B., \& Lahav, O. 1995, ApJ, 449, 446

Zaroubi, S., Hoffman, Y., \& Dekel, A. 1999, ApJ, 520, 413

Zel'dovich, Y. B. 1970, A\&A, 5, 84 